PROCEEDINGS OF THE

AMERICAN MATHEMATICAL SOCIETY

Volume 139, Number 9, September 2011, Pages 3359-3373

S 0002-9939(2011)10737-8

Article electronically published on February 10, 2011

\title{
BROWNIAN MOTION WITH VARIABLE DRIFT CAN BE SPACE FILLING
}

TONĆI ANTUNOVIĆ, YUVAL PERES, AND BRIGITTA VERMESI

(Communicated by Richard C. Bradley)

\begin{abstract}
For $d \geq 2$ let $B$ be standard $d$-dimensional Brownian motion. For any $\alpha<1 / d$ we construct an $\alpha$-Hölder continuous function $f:[0,1] \rightarrow \mathbb{R}^{d}$ so that the range of $B-f$ covers an open set. This strengthens a result of Graversen (1982) and answers a question of Le Gall (1988).
\end{abstract}

\section{INTRODUCTION}

For $d \geq 2$, let $B_{t}$ be a $d$-dimensional standard Brownian motion and $f:[0,1] \rightarrow$ $\mathbb{R}^{d}$ be a continuous function. We say $f$ is polar for $d$-dimensional Brownian motion if, for all $x$,

$$
\mathbb{P}_{x}\left\{B_{t}=f(t) \text { for some } t>0\right\}=0 .
$$

It is well known that $B_{t}$ does not hit points (see Corollary 2.24 in [12]) almost surely, hence constant functions are polar. The Cameron-Martin theorem (see Theorem 1.38 in [12] or Theorem 2.2 in Chapter 8 in 14]) tells us that for all functions $f$ in the Dirichlet space $\mathbf{D}[0,1]$ (integrals of functions in $\mathbf{L}^{2}[0,1]$ ), the path $B_{t}-f(t)$ has the same almost sure properties as the Brownian motion path. Hence functions in $\mathbf{D}[0,1]$ are polar. For dimension two Graversen proved the following.

Theorem A (Graversen [6]). For all $0<\gamma<1 / 2$, there exists a $\gamma$-Hölder continuous function $f: \mathbb{R}^{+} \rightarrow \mathbb{R}^{2}$ which is non-polar for planar Brownian motion.

Graversen also conjectured that all 1/2-Hölder continuous functions are polar and gave a partial answer to this conjecture. In [10, Le Gall proved Graversen's conjecture and gave a similar result for dimensions $d \geq 3$.

Theorem B (Le Gall [10]). Let $f: \mathbb{R}^{+} \rightarrow \mathbb{R}^{d}$ be a continuous function. Then $f$ is polar for d-dimensional Brownian motion if it satisfies one of the following conditions:

Received by the editors May 21, 2010 and, in revised form, August 11, 2010.

2010 Mathematics Subject Classification. Primary 60J65, 26A16, 26A30, 28 A80.

Key words and phrases. Brownian motion, space-filling curves, Hölder continuity.

The third author was supported by NSF Supplemental Funding DMS-0439872 to UCLA-IPAM, P.I. R. Caflisch. 
(i) $d=2$ and for all $K>0$ there exists a $\delta>0$ such that for all $s, t$ with $0 \leq s<t \leq K$ and $0<t-s<\delta$,

$$
|f(t)-f(s)| \leq\left[2(t-s) \log \log \left(\frac{1}{t-s}\right)\right]^{1 / 2},
$$

(ii) $d \geq 3$ and for each $K>0$,

$$
\lim _{\delta \rightarrow 0} \sup _{\substack{0 \leq s<t \leq K \\ t}-s<\delta} \frac{|f(t)-f(s)|}{(t-s)^{1 / d}}=0 .
$$

At the end of [10] Le Gall asked whether for each $\gamma<1 / d$ there exist $\gamma$-Hölder continuous functions which are non-polar for $d$-dimensional Brownian motion. By a simple application of Fubini's theorem, a function $f$ is non-polar if and only if the expected volume of the range of $B-f$ is positive.

In the following theorem we give a positive answer to Le Gall's question and also strengthen Graversen's Theorem.

Theorem 1.1. For $d \geq 2$, let $B_{t}$ be a d-dimensional Brownian motion. Then for any $\gamma<1 / d$, there exists a $\gamma$-Hölder continuous function $f:[0,1] \rightarrow \mathbb{R}^{d}$ for which the range of $B_{t}-f(t)$, for $t \in[0,1]$, covers an open set almost surely.

Note that in Theorem $\mathrm{B}$ the condition for polarity in higher dimensions is stronger than for 2 dimensions. We prove the following theorem which shows that a stronger condition is indeed necessary.

Theorem 1.2. For $d \geq 3$, there exists a $1 / d$-Hölder continuous function $f:[0,1] \rightarrow$ $\mathbb{R}^{d}$ that is non-polar for d-dimensional Brownian motion.

In the proof of Theorem 1.2 we take $f$ to be the standard $d$-dimensional Hilbert curve.

\section{Hilbert CURVES}

To prove Theorem 1.1 we will construct space filling curves that remain space filling even after being perturbed by Brownian motion. These curves are modifications of the standard Hilbert curve construction, which is briefly described below, for dimension two.

2.1. The standard Hilbert curve. Start with the unit square $[0,1]^{2}$. In the first iteration, subdivide the unit square into four sub-squares of side length $1 / 2$ and join their centers via three line segments. Choose a direction for the resulting path. In iteration $n+1$, take the path from iteration $n$, rescaled by $1 / 2$, and put one copy in each of the four sub-squares, rotated clockwise by $\pi / 2,0,0$ and $3 \pi / 2$ in the bottom-left, top-left, top-right and bottom-right sub-square, respectively. Then connect the four copies by adding three connection segments. The limiting curve obtained by following this iterative construction is called the Hilbert curve. Figure 1 illustrates the first three steps in the standard Hilbert curve construction, where we always traverse the path starting at the bottom-left square and ending in the bottom-right square.

An equivalent description of the Hilbert curve is as follows. For $n \geq 1$, at iteration $n$, the unit square is partitioned into a collection of $4^{n}$ squares of side length $2^{-n}$. Each of the $4^{n}$ squares is labeled by some string $a_{1}, \ldots, a_{n}$ with entries in $\{0,1,2,3\}$, which determines the order in which the squares are traversed. The 


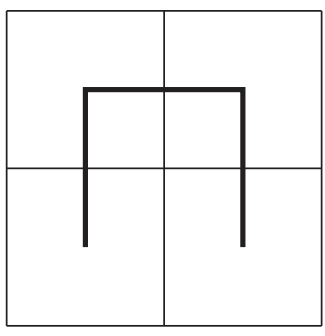

(a) $n=1$

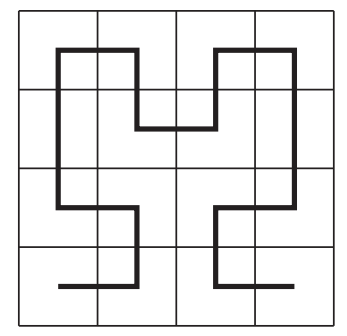

(b) $n=2$

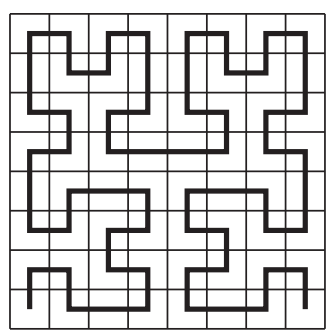

(c) $n=3$

Figure 1. The first 3 steps in the Hilbert curve construction.

main idea is to find an algorithm that recursively determines the order in which sub-squares are traversed given the order of their "ancestor" squares. Not picking the right order causes the limiting function not to be continuous. The requirement is that for any sequence $a_{1} \ldots a_{n}$ of elements in $\{0,1,2,3\}$ with $a_{n} \in\{0,1,2\}$, the square labeled by $a_{1} \ldots a_{n} 33 \ldots 3$ is very close to the square $a_{1} \ldots\left(a_{n}+1\right) 00 \ldots 0$.

Definition 2.1. The traversal order will be described by functions $h$ satisfying:

(i) $h:\{0,1,2,3\} \rightarrow\{(-1,-1),(1,-1),(1,1),(-1,1)\}$ is one-to-one,

(ii) $h(\{0,2\})=\{(-1,-1),(1,1)\}$,

(iii) $h(\{1,3\})=\{(1,-1),(-1,1)\}$.

Suppose the string $a_{1}, \ldots, a_{n}$ denotes the labeling of a square of generation $n$. The traversal order of its four sub-squares is given by the function $f_{a_{1}, \ldots, a_{n}}$, which we now construct recursively. For the empty string $\emptyset$ define $f_{\emptyset}$ as

$$
\left(f_{\emptyset}(0), f_{\emptyset}(1), f_{\emptyset}(2), f_{\emptyset}(3)\right)=((-1,-1),(-1,1),(1,1),(1,-1)) .
$$

Algorithm 1 (Standard Hilbert). Given $f_{a_{1}, \ldots, a_{n-1}}$ and $a_{n}$ define $f_{a_{1}, \ldots, a_{n}}$ to satisfy conditions in Definition 2.1 and

- if $a_{n} \in\{1,2\}$, let $f_{a_{1}, \ldots, a_{n}}=f_{a_{1}, \ldots, a_{n-1}}$,

- if $a_{n}=0$, let $f_{a_{1}, \ldots, a_{n}}(0)=f_{a_{1}, \ldots, a_{n-1}}(0), f_{a_{1}, \ldots, a_{n}}(1)=f_{a_{1}, \ldots, a_{n-1}}(3)$,

- if $a_{n}=3$, let $f_{a_{1}, \ldots, a_{n}}(0)=f_{a_{1}, \ldots, a_{n-1}}(2), f_{a_{1}, \ldots, a_{n}}(1)=f_{a_{1}, \ldots, a_{n-1}}(1)$.

Now it is not hard to give a more precise definition of the standard Hilbert curve. Let $0 . a_{1} \ldots a_{n} \ldots$ denote the 4-ary expansion of $t \in[0,1]$. We construct functions $H_{n}:[0,1] \rightarrow[0,1]^{2}$ by setting $H_{1}(t)=\left(\frac{1}{2}, \frac{1}{2}\right)+\frac{1}{4} f_{\emptyset}\left(a_{1}\right)$, and for $n \geq 2$,

$$
H_{n}(t)=H_{n-1}(t)+\left(\frac{1}{2}\right)^{n+1} f_{a_{1}, \ldots, a_{n-1}}\left(a_{n}\right) .
$$

Let $H(t)=\lim _{n \rightarrow \infty} H_{n}(t)$ be the resulting Hilbert curve. $H$ is well defined, space filling and 1/2-Hölder continuous (see Chapter 2 in [15]). Hence, by Theorem B. $H$ is polar for planar Brownian motion. Also note that the Hilbert curve is selfsimilar, through the mappings from the construction, and measure preserving with Lebesgue measure on both the unit interval and the unit square.

2.2. Generalized Hilbert curves. We now modify this construction in order to obtain curves that are space filling and Hölder continuous with the exponent slightly less than $1 / 2$, but which remain space filling after perturbation by planar 


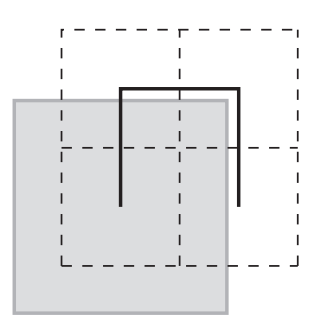

(a) $n=1$

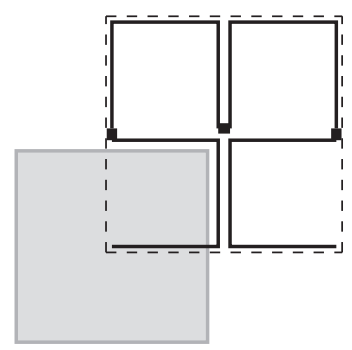

(b) $n=2$

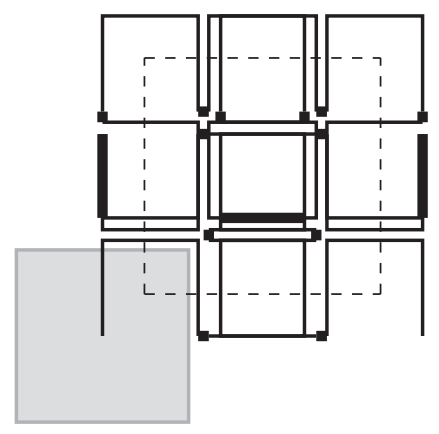

(c) $n=3$

Figure 2. First 3 steps in the new construction with $\alpha=.9$. The dashed square is the unit square, thick lines are connections between copies of lower level iterations, and the gray squares denote typical covering squares at each level.

Brownian motion. The curve will be constructed on overlapping squares, using the same algorithm as above, but with a different parametrization.

2.2.1. Main idea. Fix $\alpha>1 / 2$. At level 1, we subdivide the unit square into four sub-squares of side length $1 / 2$. Cover each of these sub-squares with a slightly larger (centered) square of side length $\alpha$, as in Figure 2, Iterate the procedure. Thus, in step $n$, each of the $4^{n}$ squares of side length $\alpha^{n}$ are covered by four overlapping squares of side length $\alpha^{n+1}$. We want to find a labeling that determines the order in which these overlapping squares are traversed, so that we obtain a continuous function in the limit. Naively, let us first try the labeling and parametrization used for the standard Hilbert curve, and to time $k / 4^{n}$ assign one of the $4^{n}$ squares of side length $\alpha^{n}$ as in the Hilbert algorithm. The curve in the first iteration is the same as the standard Hilbert curve, and the next two iterations are shown in Figure 2 .

The limiting function is space filling, but it is not continuous. To see this, first note that following the same algorithm as in the Hilbert curve construction leads to eventually starting and ending the curve outside of the unit square. In Figure 2, when $\alpha=.9$, this already happens in the third step. For a given $\alpha$, there will be an $\epsilon$ and an integer $m$ such that the starting and the ending point of the curve after $m^{\text {th }}$ iteration is at least $\epsilon$ distance, in each coordinate direction, outside the unit square. Following the standard Hilbert algorithm the endpoints of the part of the curve in the top-left sub-square should converge to the image of $1 / 2$. The same is true for the starting points of the part of curve in the top-right sub-square. However after $m^{\text {th }}$ iteration these starting points and endpoints will be (in each coordinate direction) at a distance at least $\alpha \epsilon$ from the center of the unit square. By symmetry their distance will be at least $2 \alpha \epsilon$. Therefore their limits will not be equal, which implies the discontinuity at $1 / 2$.

The main idea in our construction is the following: in each iteration, we assign three intervals of time reserved for connecting the four descendents. Choosing the right way to assign these connection times, along with the appropriate expansion factor for the squares, will allow us to obtain a Hölder continuous curve of any exponent less than $1 / 2$. The details for this construction can be found below. 
2.2.2. Scaled squares. Let $0<\alpha<1$ be given and set $\beta=\frac{2 \alpha-1}{4}$, which is negative if $\alpha<1 / 2$.

Analogous to the standard Hilbert curve construction, the set of squares at iteration $n+1$, denoted by $\mathcal{F}_{n+1}$, is constructed from $\mathcal{F}_{n}$ by taking $\mathcal{F}_{0}=\left\{[0,1]^{2}\right\}$, and if $[a, a+\delta] \times[b, b+\delta]$ is in $\mathcal{F}_{n}$, its descendents in $\mathcal{F}_{n+1}$ are

$$
[a+(i / 2-\beta) \delta, a+((i+1) / 2+\beta) \delta] \times[b+(j / 2-\beta) \delta, b+((j+1) / 2+\beta) \delta],
$$

for $i, j \in\{0,1\}$. Note that the side length of squares in $\mathcal{F}_{n}$ is equal to $\alpha^{n}$, and if $(x, y)$ is the center of a square in $\mathcal{F}_{n}$, then the centers of its four descendents are given by $(x, y)+\frac{1}{4} \alpha^{n}(i, j)$, where $i, j \in\{-1,1\}$. If $Q_{1} \in \mathcal{F}_{n}$, let $\operatorname{scale}\left(Q_{1}\right)$ denote the union of all squares $Q \in \mathcal{F}_{m}, m \geq n$, that are contained in $Q_{1}$. If $\alpha \leq 1 / 2$, then $\operatorname{scale}\left(Q_{1}\right)=Q_{1}$, and otherwise $\operatorname{scale}\left(Q_{1}\right)$ is the square with the same center as $Q_{1}$, but with side length $\left(1+\frac{2 \beta}{1-\alpha}\right) \alpha^{n}=\frac{\alpha^{n}}{2(1-\alpha)}$. In particular, if $\alpha>1 / 2$, the union of all squares, over all generations, gives the square $\left[-\frac{\beta}{1-\alpha}, 1+\frac{\beta}{1-\alpha}\right]^{2}$.

2.2.3. Generalized Hilbert curve - construction. Let $\rho<1 / 4$ and $\rho<\alpha<1$ be given. First we construct a Cantor set $C_{\rho}$ as follows. At stage 1 , divide the unit length interval into seven subintervals out of which we keep four closed intervals of length $\rho$. The collection of the intervals still kept at stage 1 is

$$
\mathrm{C}_{\rho}^{1}:=\left\{[0, \rho],\left[\frac{1-\rho}{3}, \frac{1+2 \rho}{3}\right],\left[\frac{2-2 \rho}{3}, \frac{2+\rho}{3}\right],[1-\rho, 1]\right\} .
$$

The removed open intervals will be called connection intervals. At stage $n$, each remaining closed interval of length $\rho^{n-1}$ from $C_{\rho}^{n-1}$ is divided into seven subintervals out of which four closed intervals are being kept. For example, if $\left[a, a+\rho^{n-1}\right] \in$ $\mathrm{C}_{\rho}^{n-1}$, then we have four descendents of this interval:

$$
\left\{\left[a+\frac{k}{3}(1-\rho) \rho^{n-1}, a+\rho^{n}+\frac{k}{3}(1-\rho) \rho^{n-1}\right]: k=0,1,2,3\right\} \subset \mathrm{C}_{\rho}^{n} .
$$

Let $\mathrm{C}_{\rho}=\bigcap_{n=1}^{\infty} \mathrm{C}_{\rho}^{n}$ be the resulting Cantor set. For every $t \in \mathrm{C}_{\rho}$, there exists a unique sequence $\left\{a_{n}\right\}_{n=1}^{\infty}$ with $a_{n} \in\{0,1,2,3\}$, such that $t=\frac{1-\rho}{3 \rho} \sum_{n=1}^{\infty} a_{n} \rho^{n}$. Let us call this sequence the $\rho$-expansion of $t$.

Recursively define the sequence of functions $G_{n}: C_{\rho} \rightarrow \mathbb{R}^{2}$ as follows. Abusing notation, denote the $\rho$-expansion of $t$ by $0 . a_{1} a_{2} \ldots$ and let $G_{1}(t)=\left(\frac{1}{2}, \frac{1}{2}\right)+\frac{1}{4} f_{\emptyset}\left(a_{1}\right)$. For $n \geq 2$, let

$$
G_{n}(t)=G_{n-1}(t)+\frac{\alpha^{n-1}}{4} f_{a_{1}, \ldots, a_{n-1}}\left(a_{n}\right),
$$

where the functions $f_{a_{1}, \ldots, a_{n}}$ are defined according to Algorithm 11 Let $G(t)=$ $\lim _{n \rightarrow \infty} G_{n}(t)$ for all $t \in \mathrm{C}_{\rho}$, and linearly interpolate between these values to obtain a continuous curve on $[0,1]$. It is an easy exercise to find a formula for $G(t)$ when $t \notin \mathrm{C}_{\rho}$, but we will not need it here. The function is clearly well defined and continuous. We will next show that $G$ is Hölder continuous and moreover spacefilling (its range covers an open set) for $\alpha \geq 1 / 2$.

2.2.4. Properties of generalized Hilbert curves. The range of $G_{n}$ will be exactly the set of centers of the squares in $\mathcal{F}_{n}$. For any $t \in \mathrm{C}_{\rho}$ whose $\rho$-expansion starts with $0 . a_{1} \ldots a_{n}$, denote by $D_{n}(t)$ the set of numbers in $C_{\rho}$ whose $\rho$-expansion starts with the same $n$ digits. If $G_{n}(t)$ is the center of $Q \in \mathcal{F}_{n}$, then the numbers $r \in D_{n}(t)$ will be mapped by $G_{n+1}$ into the centers of descendents of $Q$. Therefore 
$G_{n+k}(r) \in \operatorname{scale}(Q)$ for all $k \geq 0$ and $r \in D_{n}(t)$, in particular after taking limits $G(r) \in \operatorname{scale}(Q)$.

We claim $G$ is space filling if $\alpha \geq 1 / 2$; more precisely, the image of $G$ covers the entire square scale $\left([0,1]^{2}\right)=\left[-\frac{\beta}{1-\alpha}, 1+\frac{\beta}{1-\alpha}\right]^{2}$. Let $U$ be an open set that intersects the interior of the square $\left[-\frac{\beta}{1-\alpha}, 1+\frac{\beta}{1-\alpha}\right]^{2}$. Then $U$ contains a square $\operatorname{scale}(Q)$ for some $Q \in \mathcal{F}_{n}$ and for a sufficiently large $n$. As argued before, the image of $G$ will intersect $\operatorname{scale}(Q)$ and thus $U$. Therefore, the image of $G$ is dense in $\left[-\frac{\beta}{1-\alpha}\right.$, $\left.1+\frac{\beta}{1-\alpha}\right]^{2}$, and by compactness it is equal to the whole square $\left[-\frac{\beta}{1-\alpha}, 1+\frac{\beta}{1-\alpha}\right]^{2}$.

We will now compute the Hölder exponent for $G$. First it is important to note that if the interval $\left(a, a+\frac{1-4 \rho}{3} \rho^{n}\right)$ is a connection interval removed at level $(n+1)$ in the construction of $\mathrm{C}_{\rho}$, then $\left|G(a)-G\left(a+\frac{1-4 \rho}{3} \rho^{n}\right)\right|=C \alpha^{n}$, where $C=\frac{2 \beta}{1-\alpha}$ if $\alpha>1 / 2$. Also note that the endpoints of connection intervals belong to $\mathrm{C}_{\rho}$.

Let $t, s$ be in $[0,1]$, with $|t-s|<\frac{1-4 \rho}{3} \rho^{n-1}$ given. There are a few cases to be considered.

(i) If $t, s \in \mathrm{C}_{\rho}$, then they have common first $n$ digits in their $\rho$-expansions. Hence $G_{n}(t)=G_{n}(s)$, and by the discussion above there exists a constant $C_{1}$, depending only on $\alpha$, such that

$$
|G(t)-G(s)| \leq C_{1} \alpha^{n} .
$$

(ii) If $t, s \notin \mathrm{C}_{\rho}$ and they belong to the same connection interval $\left(a, a+\frac{1-4 \rho}{3} \rho^{k}\right)$ for some $k$, then

$$
|G(t)-G(s)|=\frac{\left|G(a)-G\left(a+\frac{1-4 \rho}{3} \rho^{k}\right)\right|}{\frac{1-4 \rho}{3} \rho^{k}}|t-s| \leq C_{2} \alpha^{n},
$$

for some constant $C_{2}$ which depends on $\alpha$ and $\rho$. Here we have used the fact that $\alpha / \rho>1$. The same argument holds for $s$ inside a connection interval and $t$ being one of the endpoints of this interval.

(iii) If $t \in \mathrm{C}_{\rho}$ but $s \notin \mathrm{C}_{\rho}$, and $t<s$, let $a$ be the left endpoint of the connection interval containing $s$. Then

$$
|G(t)-G(s)| \leq|G(t)-G(a)|+|G(a)-G(s)| \leq C_{1} \alpha^{n}+C_{2} \alpha^{n},
$$

from cases (i) and (ii). Similarly, we use the right endpoint of the connection interval if $t>s$.

(iv) If $t, s \notin \mathrm{C}_{\rho}$ but they belong to disjoint connection intervals, and $t<s$, we let $a$ be the right endpoint of the connection interval containing $t$. Then using cases (ii) and (iii),

$$
|G(t)-G(s)| \leq|G(t)-G(a)|+|G(a)-G(s)| \leq C_{1} \alpha^{n}+2 C_{2} \alpha^{n} .
$$

From here we see that $G$ is $\frac{\log \alpha}{\log \rho}$-Hölder continuous. Note that $\rho$ can be made arbitrarily close to $1 / 4$, and if we let $\alpha \downarrow 1 / 2$ and $\rho \uparrow 1 / 4$ simultaneously, we can get a Hölder exponent arbitrarily close to $1 / 2$.

Remark 2.2. Choosing different expansion scales for different iterations, one can construct a single function that is $\gamma$-Hölder for all $\gamma<1 / 2$. In the $n^{\text {th }}$ level of the construction we need to take $\alpha_{n}$ as the expansion factor for the squares and $\rho_{n}$ as the scaling factor for the Cantor set so that $\alpha_{n} \downarrow 1 / 2$ and $\rho_{n} \uparrow 1 / 4$. 


\section{Proof of Theorem $1.1(d=2)$}

Let $B_{t}$ be a planar Brownian motion. Almost surely, there is a (random) $\epsilon$ such that, for any $s \leq \epsilon$ and any $0 \leq t \leq 1-s$, we have $\left|B_{t+s}-B_{t}\right| \leq 3 \sqrt{s \log (1 / s)}$ (see Theorem 1.14 in [12]). The result now follows from the theorem below.

Theorem 3.1. Let $G$ be the generalized Hilbert curve constructed with $\alpha>1 / 2$. Let $h:[0,1] \rightarrow \mathbb{R}^{2}$ be a function for which there is a constant $C$ such that, for $s$ small enough and $0 \leq t \leq 1-s$, we have $|h(t+s)-h(t)| \leq C \sqrt{s \log (1 / s)}$. Then the range of the function $G+h$ covers an open set.

Proof. Pick $n_{0}$ large enough such that the assumption on $h$ holds for all $s \leq \rho^{n_{0}-1}$ and such that $\beta \alpha^{n} \geq C \sqrt{\rho^{n} n \log (1 / \rho)}$ for all $n \geq n_{0}$. Let $Q_{n}(p)$ denote a square of side length $\alpha^{n}$ centered at $p$, and recall the definition of $D_{n}(t)$ from subsection 2.2.4. By construction, for any $t \in \mathrm{C}_{\rho}$, the square $Q_{n}\left(G_{n}(t)+h(t)\right)$ is covered by the squares $Q_{n+1}\left(G_{n+1}(r)+h(t)\right)$, for $r \in D_{n}(t)$, even when each of them is shifted by no more than $\beta \alpha^{n+1}$. Now by assumption on $h$, the squares $Q_{n+1}\left(G_{n+1}(r)+h(r)\right)$, $r \in D_{n}(t)$, cover the square $Q_{n}\left(G_{n}(t)+h(t)\right)$ whenever $n \geq n_{0}$. Therefore, for all $m \geq n \geq n_{0}$ the square $Q_{n}\left(G_{n}(t)+h(t)\right)$ is covered by the squares $Q_{m}\left(G_{m}(r)+\right.$ $h(r))$, where $r$ ranges over the set $D_{n}(t)$.

Let $U$ be an open set that intersects $Q_{n_{0}}\left(G_{n_{0}}(t)+h(t)\right)$ for some $t \in \mathrm{C}_{\rho}$. By the discussion above, one can find $m$ large enough and $r \in D_{n_{0}}(t)$ such that the square $Q$ with the center at $G_{m}(r)+h(r)$ and side length equal to $C \sqrt{\rho^{m} m \log (1 / \rho)}+\frac{\alpha^{m}}{2(1-\alpha)}$ lies in $U$. If $s \in D_{m}(r)$, then $G(s)+h(s)$ lies in $Q$ and thus in $U$. This implies that the image of $G+h$ is dense in the square $Q_{n_{0}}\left(G_{n_{0}}(t)+h(t)\right)$ and, by compactness, it covers it.

Remark 3.2. As mentioned in Remark 2.2, by taking different $\alpha_{n}$ and $\rho_{n}$ at different stages in the construction of the Hilbert curve, we can obtain a generalized Hilbert curve which is $\gamma$-Hölder continuous for all $\gamma<1 / 2$. Actually such curves can be constructed in a way so that they satisfy the two-dimensional case of Theorem 1.1 . To do this we only need to ensure that Brownian motion does not destroy the overlap of squares at different levels, and the condition for this is that for large enough $n$ we have

$$
\beta_{n+1} \prod_{i=1}^{n} \alpha_{i} \geq C \sqrt{-\left(\prod_{i=1}^{n} \rho_{i}\right) \log \left(\prod_{i=1}^{n} \rho_{i}\right)}
$$

where $\beta_{n+1}=\frac{2 \alpha_{n+1}-1}{4}$. Such sequences $\alpha_{n}$ and $\rho_{n}$ can be constructed with $\alpha_{n} \downarrow 1 / 2$ and $\rho_{n} \uparrow 1 / 4$. For example, take $\alpha_{n}=\frac{e^{1 / n}}{2}$ and $\rho_{n}=\frac{e^{-1 / n}}{4}$.

\section{Construction of a Reverse Hölder Continuous drift}

In this section we describe an alternate construction of a function which, when added to standard Brownian motion, will give an almost surely space-filling curve. While its construction is slightly more complicated, this function has the advantage that it is reverse Hölder continuous.

Definition 4.1. We say that a continuous function $f: \mathbb{R} \rightarrow \mathbb{R}^{d}$ is reverse $\alpha$-Hölder continuous, for $0<\alpha<1$, if for some $C>0$ and any open interval $I$ of length $|I|$ we can find $s, t \in I$ such that $|f(s)-f(t)| \geq C|I|^{\alpha}$. 


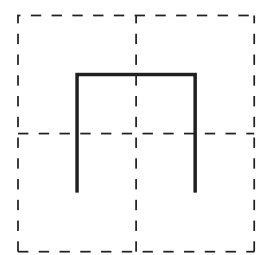

(a) $n=1$

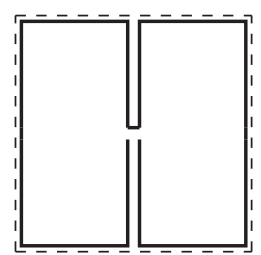

(b) $n=2$

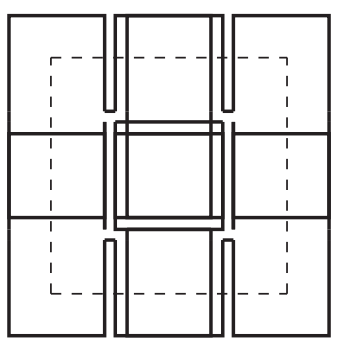

(c) $n=3$

Figure 3. First 3 steps in the new construction with $\alpha=.9$. Note that the curves start and end inside the dashed unit square.

The fact that a function is reverse Hölder continuous is closely related to the geometric properties of its graph. For example, graphs of functions which are both $\alpha$-Hölder and reverse $\alpha$-Hölder continuous (such as the function we will construct in this section) have Hausdorff dimension larger than one; see Theorem 4 in [13]. Clearly, the previously constructed generalized Hilbert curve is not reverse Hölder continuous since it is linear outside the Cantor set $\mathrm{C}_{\rho}$.

Let $\alpha>1 / 2$ be given and recall that $\beta=(2 \alpha-1) / 4$. Let $S$ be a set of positive integers such that $\sum_{i \in S} \alpha^{i}=\frac{2 \beta}{1-\alpha}$. This is the set of exceptional times when our construction deviates from the standard Hilbert algorithm. One way to construct this subset is to define $\lambda_{1}:=\frac{2 \beta}{1-\alpha}$ and, at step $i$, include in $S$ the smallest integer $j$, larger than all integers already in $S$, which has the property $\alpha^{j} \leq \lambda_{i}$ and set $\lambda_{i+1}=\lambda_{i}-\alpha^{j}$. We call the string $a_{1}, \ldots, a_{n} 0$-reverse (3-reverse) if $a_{1}, \ldots, a_{n}$ ends in exactly $k$ 's's ( $k 3$ 's) for some $k$ with $k+1 \in S$. This means that $a_{n-k+1}=\cdots=a_{n}=0 \neq a_{n-k}$, and similarly for 3's. For a string that ends in several consecutive 0's (3's), we define its prestring to be the string obtained by deleting the maximal block of consecutive 0's (3's) at the end.

Algorithm 2 (Alternate generalized Hilbert). Define $f_{\emptyset}$ by (2.1). Let $a_{1}, \ldots, a_{n}$ be given and let $a_{1}, \ldots, a_{m}$ be its prestring. Given $f_{a_{1}, \ldots, a_{n-1}}$ and $a_{n}$ define $f_{a_{1}, \ldots, a_{n}}$ to satisfy the conditions in Definition 2.1 and

- if $a_{n} \in\{1,2\}$, let $f_{a_{1}, \ldots a_{n}}=f_{a_{1}, \ldots, a_{n-1}}$;

- if $a_{n}=0$, let $f_{a_{1}, \ldots, a_{n}}(1)=f_{a_{1}, \ldots, a_{n-1}}(3)$ and

- if $a_{1}, \ldots, a_{n}$ is 0-reverse, let $f_{a_{1}, \ldots, a_{n}}(0)=f_{a_{1}, \ldots, a_{m}}(2)$,

- if $a_{1}, \ldots, a_{n}$ is not 0 -reverse, let $f_{a_{1}, \ldots, a_{n}}(0)=f_{a_{1}, \ldots, a_{m}}(0)$;

- if $a_{n}=3$, let $f_{a_{1}, \ldots, a_{n}}(0)=f_{a_{1}, \ldots, a_{n-1}}(2)$ and

- if $a_{1}, \ldots, a_{n}$ is 3 -reverse, let $f_{a_{1}, \ldots, a_{n}}(1)=f_{a_{1}, \ldots, a_{m}}(3)$,

- if $a_{1}, \ldots, a_{n}$ is not 3 -reverse, let $f_{a_{1}, \ldots, a_{n}}(1)=f_{a_{1}, \ldots, a_{m}}(1)$.

For $n \geq 1$ let $\mathbb{Q}_{n}=\left\{k 4^{-n}: k \in \mathbb{Z}, 0 \leq k \leq 4^{n}\right\}$. For every $0 \leq t \leq 1$ consider its 4 -ary expansion $t=0 . a_{1} a_{2} \ldots$ Now define $\widetilde{G}_{0}$ to be the constant function mapping into the center of the unit square $[0,1]^{2}$, and for $n \geq 1$, let the sequence of functions $\widetilde{G}_{n}$ be given by recursion $(2.3)$. This is a Cauchy sequence of functions and so finally defines $\widetilde{G}$ as their limit. The space-filling property of this function is obvious. 
Now we prove that the constructed function is both $\frac{\log (1 / \alpha)}{\log 4}$-Hölder and reverse $\frac{\log (1 / \alpha)}{\log 4}$-Hölder continuous. For the reverse Hölder continuity notice that any interval $I \subset[0,1]$ contains a 4 -ary interval $J$, of the form $\left[k 4^{-n},(k+1) 4^{-n}\right]$, of length at least $|I| / 8$. Moreover, any 4 -ary interval $J$ of length $4^{-n}$ is mapped by $\widetilde{G}$ onto a square of side length $\frac{\alpha^{n}}{2(1-\alpha)}=\frac{1}{2(1-\alpha)}|J|^{\log (1 / \alpha) / \log 4}$. Pick $s, t \in J$ so that $\widetilde{G}(s)$ and $\widetilde{G}(t)$ are vertices of one side of the square $\widetilde{G}(J)$. We see that

$$
|\widetilde{G}(s)-\widetilde{G}(t)|=\frac{1}{2(1-\alpha)}|J|^{\log (1 / \alpha) / \log 4} \geq \frac{\alpha^{3 / 2}}{2(1-\alpha)}|I|^{\log (1 / \alpha) / \log 4} .
$$

If $s \in \mathbb{Q}_{n}$ and $t$ is any number whose 4 -ary expansion agrees in the first $n$ digits with $s$, then $|\widetilde{G}(t)-\widetilde{G}(s)| \leq \frac{\alpha^{n}}{2(1-\alpha)}$. Take any $0 \leq t_{1}<t_{2} \leq 1$, and $n$ such that $t_{2}-t_{1} \leq 4^{-n}$ and $q_{1}, q_{2} \in \mathbb{Q}_{n}$ such that $0 \leq t_{i}-q_{i} \leq 4^{-n}$, for $i=1,2$. Then we have $\left|\widetilde{G}\left(t_{i}\right)-\widetilde{G}\left(q_{i}\right)\right| \leq \frac{\alpha^{n}}{2(1-\alpha)}$ for $i=1,2$ and $\left|\widetilde{G}\left(t_{2}\right)-\widetilde{G}\left(t_{1}\right)\right| \leq \frac{\alpha^{n}}{1-\alpha}+\left|\widetilde{G}\left(q_{1}\right)-\widetilde{G}\left(q_{2}\right)\right|$. Since either $q_{1}=q_{2}$ or $q_{2}=q_{1}+4^{-n}$ we see that it suffices to prove the Hölder continuity for points in $\bigcup_{n} \mathbb{Q}_{n}$.

Let $s, t \in \mathbb{Q}_{n}$ with $t=s+4^{-n}$. If the 4 -ary expansion of $s$ does not end in 3, then $s$ and $t$ have equal first $n-1$ digits, and so $|\widetilde{G}(t)-\widetilde{G}(s)| \leq \frac{1}{\alpha(1-\alpha)} \alpha^{n}$ and we are done. Otherwise let the 4-ary expansion of $s$ be given by $s=0 . a_{1} \ldots a_{m} 3 \ldots 3$ with $k=n-m 3$ 's in the end and $a_{m} \neq 3$. Then obviously $t$ can be written in base 4 as $t=0 . a_{1} \ldots a_{m-1}\left(a_{m}+1\right) 0 \ldots 0$. Define $s_{l}=0 . a_{1} \ldots a_{m} 3 \ldots 3$ with $l 3$ 's in the end, so in particular $s_{k}=s$. Without loss of generality assume $f_{a_{1}, \ldots, a_{m-1}}=f_{\emptyset}$. Denote the point $\widetilde{G}_{m-1}(t)=\widetilde{G}_{m-1}(s)$ by $p$. Let us verify the claim for $a_{m}=1$ since cases $a_{m} \in\{0,2\}$ are similar.

If $a_{m}=1$, then $f_{a_{1}, \ldots, a_{m}}=f_{\emptyset}=f_{a_{1}, \ldots,\left(a_{m}+1\right)}$ and, relative to $p$, the coordinates of $\widetilde{G}_{m}(s)$ and $\widetilde{G}_{m}(t)$ are $\left(-\frac{\alpha^{m-1}}{4}, \frac{\alpha^{m-1}}{4}\right)$ and $\left(\frac{\alpha^{m-1}}{4}, \frac{\alpha^{m-1}}{4}\right)$, respectively. Now, for any $l>0$, the point $\widetilde{G}_{m+l}\left(s_{l}\right)$ is constructed from $\widetilde{G}_{m+l-1}\left(s_{l-1}\right)$ by shifting it by $\frac{\sqrt{2}}{4} \alpha^{m+l-1}$ in the lower right direction, unless $l \in S$ when we have to shift the point by the same distance in the upper left direction. From the definition of $S$ it is clear that $\widetilde{G}_{m+l}\left(s_{l}\right)$ will converge to $p$ as $l \rightarrow \infty$ and moreover that $\left|\widetilde{G}_{m+l}\left(s_{l}\right)-p\right| \leq \sqrt{2} \alpha^{m} \sum_{j \geq l} \alpha^{j}=\frac{\sqrt{2}}{1-\alpha} \alpha^{m+l}$. In particular for $l=k$ this implies that

$|\widetilde{G}(s)-p| \leq\left|\widetilde{G}(s)-\widetilde{G}_{n}(s)\right|+\left|\widetilde{G}_{n}(s)-p\right| \leq \frac{1+2 \sqrt{2}}{2(1-\alpha)} \alpha^{n}=\frac{1+2 \sqrt{2}}{2(1-\alpha)}(t-s)^{\log (1 / \alpha) / \log 4}$.

Similarly, we argue that $\widetilde{G}_{m+l}(t)$ also converges to $p$ which implies $\widetilde{G}(t)=p$, and plugging this into (4.1) gives the wanted bound.

Adding $\tilde{G}$ to planar Brownian motion results in a space-filling process. The proof of this fact is analogous to the proof of Theorem 1.1] when $d=2$; hence we do not include it here. In $d$ dimensions $(d \geq 3)$, one can also construct a continuous curve with Hölder exponent arbitrarily close to $1 / d$, with the same properties as $\tilde{G}$, by changing the traversal algorithm given in the $d$-dimensional Hilbert curve construction. Such a higher-dimensional curve, analogous to $\tilde{G}$, can be described using a modification of Butz's algorithm from [4]. 


\section{Proof of Theorem $1.1(d>2)$}

To prove Theorem 1.1 in dimensions higher than 2, we construct generalized Hilbert curves in the same way as in two dimensions. There are algorithms that generate standard $d$-dimensional Hilbert curves in dimensions higher than two, which can be realized through the labeling algorithm we described for two dimensions. One example is the Butz algorithm; see [3] and 4. These algorithms are completely determined by functions $f_{a_{1}, \ldots, a_{n}}$ where $a_{i} \in\left\{0,1, \ldots, 2^{d}-1\right\}$. Throughout this section $f_{a_{1}, \ldots, a_{n}}$ will stand for these functions. The Hilbert curve is defined as the limit of the functions defined recursively by formulae analogous to (2.2). As in the two-dimensional case, the Hilbert curve is again measure preserving. Here we do not state the explicit algorithms for generating functions $f_{a_{1}, \ldots, a_{n}}$, as they are relatively complicated. See also [15] for a geometric description of space-filling curves in higher dimensions.

Let $\rho<2^{-d}$ be given and define $\mathrm{C}_{\rho, d}$ by iteratively removing, at step $n+1$, exactly $2^{\text {nd }}\left(2^{d}-1\right)$ (open) connection intervals of length $\frac{1-\left(2^{d}\right) \rho}{2^{d}-1} \rho^{n}$ and keeping $2^{(n+1) d}$ intervals of length $\rho^{n+1}$. As in the 2 -dimensional case, every $t \in \mathrm{C}_{\rho, d}$ has a unique representation

$$
t=K \sum_{n=1}^{\infty} a_{n} \rho^{n}, \quad a_{n} \in\left\{0,1 \ldots, 2^{d}-1\right\},
$$

with $K=\frac{1-\rho}{\left(2^{d}-1\right) \rho}$. We will call $0 . a_{1} a_{2} \ldots$ the $(\rho, d)-$ expansion of $t$. Fix an $\alpha>1 / 2$ and define functions $G_{0}^{d}$ to be the constant function mapping to the center of the unit cube $[0,1]^{d}$ and $G_{n}^{d}$ recursively by (2.3), with the understanding that the traversal algorithm and hence the functions $f_{a_{1}, \ldots, a_{n}}$ correspond to the $d$-dimensional Hilbert curve. Then define $G^{d}$ as the limit of functions $G_{n}^{d}$. Again, for $t \notin \mathrm{C}_{\rho, d}$, the values $G^{d}(t)$ are defined by interpolation. The resulting curve is clearly well-defined and continuous. Hölder continuity and the space covering property for $G^{d}$ follow by the same argument as in the two-dimensional case. The Hölder exponent is again $\frac{\log \alpha}{\log \rho}$. We leave the details to the reader. Taking $\rho$ close to $1 / 2^{d}$ and $\alpha$ close to $1 / 2$ leads to continuous functions with Hölder exponents arbitrarily close to $1 / d$.

Proof of Theorem $1.1(d>2)$. As in the two-dimensional case, there exists a constant $C>0$ such that, almost surely, for every small enough $s>0$ and all $0 \leq t \leq 1-s$,

$$
\left|B_{t+s}-B_{t}\right| \leq C \sqrt{s \log (1 / s)} .
$$

Suppose $n_{0}$ is large enough such that condition (5.1) holds for $s \leq \rho^{n_{0}-1}$ and such that, for all $n \geq n_{0}$,

$$
\beta \alpha^{n} \geq C \sqrt{\rho^{n} n \log (1 / \rho)} .
$$

For $t \in \mathrm{C}_{\rho, d}$ with $(\rho, d)$-expansion starting with $0 . a_{1} \ldots a_{n}$ define $D_{n}(t)$ as the set of numbers $r \in \mathrm{C}_{\rho, d}$ whose $(\rho, d)$-expansion starts with the same $n$ digits. Take the box $Q_{n_{0}}$ of side length $\alpha^{n_{0}}$ centered at $B_{t}-G_{n_{0}}^{d}(t)$. Just as in the proof of Theorem 3.1. it is easy to see that the $2^{d}$ boxes of side length $\alpha^{n_{0}+1}$ centered at $B_{r}-G_{n_{0}+1}^{d}(r)$, for $r \in D_{n_{0}}(t)$, cover $Q_{n_{0}}$. Iterating, we see that for all $m \geq 1$, the box $Q_{n_{0}}$ is covered by the $2^{m d}$ boxes of side length $\alpha^{n_{0}+m}$ centered at $B_{r}-G_{n_{0}+m}^{d}(r)$, for $r \in D_{n_{0}}(t)$. Then given an arbitrary point $x \in Q_{n_{0}}$, one can find a sequence of 
boxes $\left\{Q_{n_{0}+m}\right\}_{m \geq 1}$, of side length $\alpha^{n_{0}+m}$, centered as above, that contain $x$. From here it easily follows that $x$ is in the image of $B-G^{d}$, which completes the proof.

Remark 5.1. Let $G$ be a generalized $d$-dimensional Hilbert curve with $\alpha<1 / 2$. These cases are also interesting since $B-G$ is not space filling, and moreover, for $\rho<\alpha^{2}$, we can compute the Hausdorff dimension of its range to be exactly $\max \left(d \frac{\log 2}{\log (1 / \alpha)}, 2\right)$. To prove this, notice that since $[0,1] \backslash C_{\rho, d}$ is a countable union of disjoint intervals, Hausdorff dimension of $(B-G)\left([0,1] \backslash C_{\rho, d}\right)$ is equal to 2 . Therefore it is enough to prove that $(B-G)\left(\mathrm{C}_{\rho, d}\right)$ has Hausdorff dimension equal to $d \frac{\log 2}{\log (1 / \alpha)}$. Denote with $Q_{n}(p)$ the cube of side length $\alpha^{n}$, centered at $p$. It is easy to check that there is a $\gamma>0$ such that, for all $t \in \mathrm{C}_{\rho, d}$, the cubes $Q_{n+1}\left(B_{t}-G_{n+1}(r)\right)$ for $r \in D_{n}(t)$ will be disjoint and contained in the cube $Q_{n}\left(B_{t}-G_{n}(t)\right)$, even if each of the smaller cubes is shifted by no more than $\gamma \alpha^{n+1}$. Now knowing the modulus of continuity of Brownian motion and using the same argument as in the proof of Theorem 1.1 (and the fact that $\rho<\alpha^{2}$ ), we see that for some (random) $n_{0}$ and all $n \geq n_{0}$ the cubes $Q_{n+1}\left(B_{r}-G_{n+1}(r)\right)$ for $r \in D_{n}(t)$ will be disjoint and contained in the cube $Q_{n}\left(B_{t}-G_{n}(t)\right)$, even after being shifted by no more than $\gamma \alpha^{n+1} / 2$. As a consequence, for any $m \geq n_{0}$ and $r \in D_{m}(t)$ every two of the points $B_{r}-G_{m}(r)$ are at a distance greater than $\gamma \alpha^{m} / 2$. This immediately implies that the Hausdorff dimension of the image $(B-G)\left(C_{\rho, d}\right)$ is almost surely bounded by $d \frac{\log 2}{\log (1 / \alpha)}$. For the lower bound we can use the Mass Distribution Principle (see 4.2, Chapter 4 in [5]). For a fixed $t \in \mathrm{C}_{\rho, d}$ we define a measure on the part of $(B-G)\left(\mathrm{C}_{\rho, d}\right)$ which is contained in $Q_{n_{0}}\left(B_{t}-G_{n_{0}}(t)\right)$. The measure is simply defined by assigning mass $2^{-k d}$ to each of the disjoint cubes $Q_{n_{0}+k}\left(B_{s}-G_{n_{0}+k}(s)\right)$ for $s \in D_{n_{0}}(t)$. Then any ball of radius $\gamma \alpha^{n_{0}+m} / 2$ is assigned no more than $2^{-m d}$ mass, and the claim follows by the Mass Distribution Principle.

Therefore, for any $2<\lambda<d$ and any $\kappa<1 / \lambda$, we can find a generalized $d$ dimensional Hilbert curve which is $\kappa$-Hölder continuous and such that almost surely the range of Brownian motion with this drift has Hausdorff dimension equal to $\lambda$, by taking $\alpha=\rho^{\kappa}=2^{-d / \lambda}$ in our construction.

\section{Proof of Theorem 1.2}

We will prove Theorem 1.2 by showing that for $d>2$ the range of $d$-dimensional Brownian motion with drift given by the standard $d$-dimensional Hilbert curve hits points with positive probability. The key ingredient in the proof is the following lemma which shows that, for any $\alpha>1 / d$, the Hilbert curve satisfies the reverse Hölder inequality for all points from a set of large measure.

Lemma 6.1. For $d \geq 3$, let $H:[0,1] \rightarrow[0,1]^{d}$ denote the standard d-dimensional Hilbert curve. Then for any $\alpha>1 / d$ and any $\epsilon>0$, there is a set $S \subset[0,1]$ of Lebesgue measure greater than $1-\epsilon$ and $a C>0$ such that $|H(s)-H(t)| \geq C|s-t|^{\alpha}$ for all $s, t \in S$.

Proof. As usual $\mathcal{F}_{n}$ is the set of dyadic cubes used in the construction of the Hilbert curve. Define $W_{n}:=\bigcup_{Q \in \mathcal{F}_{n}} \partial Q$. For $\delta>0$ we define $B\left(W_{n}, \delta\right)$ as the set of points in $[0,1]^{d}$ at a distance no more than $\delta$ from $W_{n}$.

First we show that the volume of the set $B\left(W_{n}, 2^{-n-m}\right)$ is bounded from above by $2 d 2^{-m}$. We prove this by induction. For $n=0$, this is trivial since $W_{0}$ is just the boundary of the original cube whose $(d-1)$-dimensional volume is equal to $2 d$. 
When $m=0$, the claim is also trivial since $B\left(W_{n}, 2^{-n-m}\right)$ is equal to the whole original cube. In the general case, notice that the intersection of $B\left(W_{n}, 2^{-n-m}\right)$ with a cube from $\mathcal{F}_{1}$ is equal to $B\left(W_{n-1}, 2^{-n-m+1}\right)$, scaled by a factor of $1 / 2$ and translated accordingly. The induction assumption implies that the volume of this intersection is bounded from above by $2 d 2^{-d} 2^{-m}$. Summing over all cubes in $\mathcal{F}_{1}$ proves the claim.

For $c>0$ define $K:=\bigcup_{n \geq 0} B\left(W_{n}, c 2^{-n d \alpha}\right)$ and set $S:=H^{-1}\left(K^{c}\right)$. Since the volume of $K$ is less than $c \sum_{n>0} 2^{-n(d \alpha-1)}$ and $H$ is measure preserving, we can choose the constant $c$ to make the Lebesgue measure of $S$ arbitrarily close to 1 .

To check that $S$ satisfies the desired property, take $s, t \in S$ and let $n$ be the largest integer so that $H(s)$ and $H(t)$ are contained in the same box in $\mathcal{F}_{n-1}$. Then $|s-t| \leq 2^{-d(n-1)}$, but $|H(s)-H(t)| \geq 2 c 2^{-n d \alpha}$, since $H(s)$ and $H(t)$ are in $K^{c}$ and inside different boxes in $\mathcal{F}_{n}$. This proves the claim with $C=2^{1-d \alpha} c$.

Proof of Theorem 1.2. Let $1 / d<\alpha<\frac{3}{2(d+1)} \leq 1 / 2$ be given and let $H$ denote the regular $d$-dimensional Hilbert curve. Let $J$ be a set of positive measure with $|H(s)-H(t)| \geq C|s-t|^{\alpha}$ for all $s, t \in J$, for some constant $C>0$, as in Lemma 6.1. Without loss of generality we can assume $J$ is closed.

Fix $\delta>0$ and $x \in \mathbb{R}^{d}$, and set $J_{\delta}=[\delta, 1] \cap J$. We let $T_{\epsilon}$ be the amount of time in $J_{\delta}$ that the range of function $t \mapsto B_{t}-H(t)$ spends in $\mathcal{B}_{\epsilon}:=B(x, \epsilon)$, the ball of radius $\epsilon$ around $x$. We will apply the second moment method to $T_{\epsilon}$. Using Fubini and the fact that the density of $d$-dimensional Brownian motion is bounded from below on $\mathcal{B}_{\epsilon}$ when $t \geq \delta$, we have

$$
\mathbb{E}\left(T_{\epsilon}\right)=\int_{J_{\delta}} \mathbb{P}\left(B_{t}-H(t) \in \mathcal{B}_{\epsilon}\right) d t \geq b \epsilon^{d},
$$

for some constant $b$ depending only on $\delta$ and $d$. To find the second moment of $T_{\epsilon}$, apply Fubini to obtain

$$
\mathbb{E}\left(T_{\epsilon}^{2}\right)=2 \int_{J_{\delta}} \mathbb{P}\left(B_{s}-H(s) \in \mathcal{B}_{\epsilon}\right) \int_{J_{\delta} \cap[s, 1]} \mathbb{P}\left(B_{t}-H(t) \in \mathcal{B}_{\epsilon} \mid B_{s}-H(s) \in \mathcal{B}_{\epsilon}\right) d t d s .
$$

By bounding density for $s \in J_{\delta}$ we have $\mathbb{P}\left(B_{s}-H(s) \in \mathcal{B}_{\epsilon}\right) \leq C_{1} v_{d} \epsilon^{d}$, where $C_{1}$ depends on $\delta$ and $v_{d}$ denotes the volume of the unit ball in $d$ dimensions. Thus we only need to estimate the inner integral in (6.2). Define the set

$$
\tilde{J}_{s}=\{t \in J: t \geq s,|H(t)-H(s)|>3 \epsilon\} .
$$

The inner integral in (6.2) can be separated into three parts. Let

$$
\begin{aligned}
& A_{1}:=\int_{[s, 1] \cap\left(J_{\delta} \backslash \tilde{J}_{s}\right)} \mathbb{P}\left(B_{t}-H(t) \in \mathcal{B}_{\epsilon} \mid B_{s}-H(s) \in \mathcal{B}_{\epsilon}\right) d t \\
& A_{2}:=\int_{\left[s+(4 \epsilon / C)^{1 / \alpha}, 1\right] \cap J_{\delta}} \mathbb{P}\left(B_{t}-H(t) \in \mathcal{B}_{\epsilon} \mid B_{s}-H(s) \in \mathcal{B}_{\epsilon}\right) d t \\
& A_{3}:=\int_{\left[s, s+(4 \epsilon / C)^{1 / \alpha}\right] \cap J_{\delta} \cap \tilde{J}_{s}} \mathbb{P}\left(B_{t}-H(t) \in \mathcal{B}_{\epsilon} \mid B_{s}-H(s) \in \mathcal{B}_{\epsilon}\right) d t .
\end{aligned}
$$

Because $H$ is measure preserving, the Lebesgue measure of $J \backslash \tilde{J}_{s}$ can be bounded above by $v_{d} 3^{d} \epsilon^{d}$. Thus, using a trivial bound for the probability inside the integral, we have

$$
A_{1} \leq v_{d} 3^{d} \epsilon^{d}
$$


For the second integral, notice that the distance between the balls $B(x+H(s), \epsilon)$ and $B(x+H(t), \epsilon)$ is at least $C(t-s)^{\alpha}-2 \epsilon \geq C(t-s)^{\alpha} / 2$. Therefore, by bounding the density,

$$
A_{2} \leq \int_{s+(4 \epsilon / C)^{1 / \alpha}}^{1} v_{d} \epsilon^{d} \frac{\exp \left\{-C^{2}(t-s)^{2 \alpha-1} / 8\right\}}{(2 \pi(t-s))^{d / 2}} d t<C_{2} v_{d} \epsilon^{d},
$$

for some constant $C_{2}$, where we use the fact that $\lim _{r \downarrow 0} \exp \left(-r^{p}\right) r^{q}<\infty$ for $p<0$ and any $q$. Finally, if both $B_{s}-H(s)$ and $B_{t}-H(t)$ are in $\mathcal{B}_{\epsilon}$ and $t \in \tilde{J}_{s}$, then it must hold that $\left|B_{t}-B_{s}\right| \geq \epsilon$, and so

$$
\begin{aligned}
A_{3} \leq \int_{s}^{s+(4 \epsilon / C)^{1 / \alpha}} \mathbb{P}( & \left.\frac{\left|B_{t}-B_{s}\right|}{\sqrt{t-s}} \geq \frac{\epsilon}{\sqrt{t-s}}\right) d t \\
& \leq \int_{s}^{s+(4 \epsilon / C)^{1 / \alpha}} C^{\prime} \frac{\sqrt{t-s}}{\epsilon} d t=\frac{2 C^{\prime}}{3}\left(\frac{4}{C}\right)^{3 /(2 \alpha)} \epsilon^{3 /(2 \alpha)-1},
\end{aligned}
$$

where the second inequality follows by Markov inequality. Since $\alpha<\frac{3}{2(d+1)}$, it follows that $A_{3} \leq C_{3} \epsilon^{d}$, for some constant $C_{3}$.

Now summing all the bounds, we have that $\mathbb{E}\left(T_{\epsilon}^{2}\right) \leq K \epsilon^{2 d}$, for some $K>0$, and by the Paley-Zygmund inequality we obtain

$$
\mathbb{P}\left(B_{t}-H(t)=x, \text { for some } t\right) \geq \lim _{\epsilon \rightarrow 0} \mathbb{P}\left(T_{\epsilon}>0\right) \geq \lim _{\epsilon \rightarrow 0} \frac{\mathbb{E}\left(T_{\epsilon}\right)^{2}}{\mathbb{E}\left(T_{\epsilon}^{2}\right)} \geq \frac{b^{2}}{K} .
$$

Theorem 1.2 tells us that even after the Brownian perturbation the standard Hilbert curve has positive volume. It would be interesting to see whether it also remains space filling.

Remark 6.2. We give an alternate proof of Graversen's result, where for any $\alpha<$ $1 / 2$ there exists an $\alpha$-Hölder continuous function $f: \mathbb{R}^{+} \rightarrow \mathbb{R}^{2}$ which is not polar for two-dimensional Brownian motion. The function $f$ in our example will vary only in one coordinate. Recall that the fractional Brownian motion with Hurst index $0<H<1$ is a continuous centered Gaussian process $B^{(H)}: \mathbb{R}^{+} \rightarrow \mathbb{R}$ with $\mathbb{E}\left[\left(B^{(H)}(t)-B^{(H)}(s)\right)^{2}\right]=|t-s|^{2 H}$, and for $H=1 / 2$ it is just a one-dimensional Brownian motion. It is well known that the process $B^{(H)}$ is $\alpha$-Hölder continuous for any $\alpha<H$ almost surely. For this and other basic results on fractional Brownian motion see Chapter 1 in [1]. The Hausdorff dimension of the level sets of $B^{(H)}(t)$ is, almost surely, bounded from below by $1-H$; see Theorems 4 and 5 in Chapter 18 of [8] (actually, equality holds here, but we will need only the lower bound). The same arguments show that, for an independent Brownian motion $B(t)$, the Hausdorff dimension of any level sets of $B(t)-B^{(H)}(t)$ is also bounded from below by $1-H$ almost surely (see also Proposition 5.1 and Section 7.2 in [2]). Moreover, it is well known that, for a standard one-dimensional Brownian motion and any $x \in \mathbb{R}$, any set of Hausdorff dimension strictly greater than $1 / 2$ is intersected by the level set $\{t: B(t)=x\}$ with positive probability. A standard way to prove this fact is to show that the zero set of a one-dimensional Brownian motion is the image of a 1/2-stable subordinator, a result that goes back to Lévy [11] (see also Section 6.2 D in [9]), and then to refer to a standard result in potential theory of subordinators (Lemma 2 in [7]). Assume now that $\left(B_{1}(t), B_{2}(t)\right)$ is a standard two-dimensional Brownian motion independent of the fractional Brownian motion $B^{(H)}(t)$, where $H<1 / 2$. 
By our discussion above and independence, for any $\left(x_{1}, x_{2}\right) \in \mathbb{R}^{2}$, conditioned on the realization of the set $\left\{t: B_{1}(t)-B^{(H)}(t)=x_{1}\right\}$, it will be intersected by $\left\{t: B_{2}(t)=x_{2}\right\}$ with positive probability. This means that, for $H<1 / 2$, the process $\left(B^{(H)}(t), 0\right)$ is almost surely non-polar for $\left(B_{1}(t), B_{2}(t)\right)$. Since $\left(B^{(H)}(t), 0\right)$ is $\alpha$-Hölder continuous for any $\alpha<H$, the claim follows.

Remark 6.3. We end the paper by giving a proof, different from the one in [10], of Le Gall's result that any function satisfying (1.2) is polar. It is based on the fact that images of such functions have zero volume. In the following $|S|$ will denote the diameter of a set $S$. If $f: \mathbb{R}^{+} \rightarrow \mathbb{R}^{d}$ satisfies (1.2), then for any $\epsilon>0$ we can find a $\delta>0$ such that for any set $S \subset \mathbb{R}$ of diameter $|S| \leq \delta$ we have $|f(S)| \leq \epsilon|S|^{1 / d}$. Covering an interval $I$ of length $l$ by intervals $I_{1}, \ldots I_{n}$ of length $l / n$ for $n$ large enough, we obtain a cover $f\left(I_{j}\right), 1 \leq j \leq n$, for the image $f(I)$ which satisfies $\sum_{j}\left|f\left(I_{j}\right)\right|^{d} \leq \epsilon l$. Therefore the $d$-dimensional Hausdorff measure of $f(I)$ is zero. Since Brownian motion is almost surely $\alpha$-Hölder continuous for any $\alpha<1 / 2$, we have that $B-f$ satisfies the same condition (1.2) and therefore its image has volume zero almost surely. Now the result follows by an application of Fubini and standard arguments.

\section{ACKNOWLEDGMENTS}

The first and third authors would like to thank Microsoft Research and the University of Washington, where this research was completed.

\section{REFERENCES}

[1] Francesca Biagini, Yaozhong Hu, Bernt Øksendal, and Tusheng Zhang, Stochastic calculus for fractional Brownian motion and applications, Probability and its Applications (New York), Springer-Verlag London Ltd., London, 2008. MR2387368 (2010a:60145)

[2] Brahim Boufoussi, Marco Dozzi, and Raby Guerbaz, Path properties of a class of locally asymptotically self similar processes, Electron. J. Probab. 13 (2008), no. 29, 898-921. MR 2413288 (2009e:60088)

[3] A. R. Butz, Alternative algorithm for Hilbert's space-filling curve, IEEE Trans. Comput. 20 (1971), no. 4, 424-426.

[4] Arthur R. Butz, Convergence with Hilbert's space filling curve, J. Comput. System Sci. 3 (1969), 128-146. MR0246631 (39:7935)

[5] Kenneth Falconer, Fractal geometry, second ed., John Wiley \& Sons Inc., Hoboken, NJ, 2003, Mathematical foundations and applications. MR2118797 (2006b:28001)

[6] S. E. Graversen, "Polar"-functions for Brownian motion, Z. Wahrsch. Verw. Gebiete 61 (1982), no. 2, 261-270. MR675615 (84j:60090)

[7] John Hawkes, On the Hausdorff dimension of the intersection of the range of a stable process with a Borel set, Z. Wahrscheinlichkeitstheorie und Verw. Gebiete 19 (1971), 90-102. MR0292165 (45:1252)

[8] Jean-Pierre Kahane, Some random series of functions, second ed., Cambridge Studies in Advanced Mathematics, vol. 5, Cambridge University Press, Cambridge, 1985. MR 833073 (87m:60119)

[9] Ioannis Karatzas and Steven E. Shreve, Brownian motion and stochastic calculus, second ed., Graduate Texts in Mathematics, vol. 113, Springer-Verlag, New York, 1991. MR 1121940 (92h:60127)

[10] Jean-François Le Gall, Sur les fonctions polaires pour le mouvement brownien, Séminaire de Probabilités, XXII, Lecture Notes in Math., vol. 1321, Springer, Berlin, 1988, pp. 186-189. MR960526 (90a:60146)

[11] Paul Lévy, Sur certains processus stochastiques homogènes, Compositio Math. 7 (1939), 283339. MR0000919 (1:150a) 
[12] Y. Peres and P. Mörters, Brownian motion, Cambridge Series in Statistical and Probabilistic Mathematics, Cambridge University Press, 2010. MR 2604525

[13] F. Przytycki and M. Urbański, On the Hausdorff dimension of some fractal sets, Studia Math. 93 (1989), no. 2, 155-186. MR.1002918 (90f:28006)

[14] Daniel Revuz and Marc Yor, Continuous martingales and Brownian motion, third ed., Grundlehren der Mathematischen Wissenschaften [Fundamental Principles of Mathematical Sciences], vol. 293, Springer-Verlag, Berlin, 1999. MR,1725357 (2000h:60050)

[15] Hans Sagan, Space-filling curves, Universitext, Springer-Verlag, New York, 1994. MR.1299533 (95h:00001)

Department of Mathematics, University of California, Berkeley, Berkeley, CaliFORNIA 94720

E-mail address: tantun@math.berkeley.edu

Theory Group, Microsoft Research, Redmond, Washington 98052

E-mail address: peres@microsoft.com

Department of Mathematics, University of Washington, Box 354322, Seattle, Washington 98195 - And - Institute for Pure and Applied Mathematics, 460 Portola Plaza, Los Angeles, California 90095

E-mail address: bvermesi@math.washington.edu 